\title{
Hesitation and Error - Does Product Placement in an Emergency Department Influence Hand Hygiene Performance?
}

Background: Existing research has consistently demonstrated poor compliance by healthcare workers with hand hygiene standards. This study examined the extent to which incorrect hand hygiene occurred as a result of the inability to easily distinguish between different hand hygiene solutions placed at washbasins.

Methods: A direct observational method was employed using ceiling-mounted, motionactivated video camera surveillance in a tertiary referral emergency department (ED) in Australia. Data from a 24-hour period on day 10 of the recordings were collected into the Hand Hygiene - Technique Observation Tool (HH-TOT) based on Feldman's Criteria as modified by Larson and Lusk.

Results: A total of 459 episodes of hand hygiene were recorded by six video cameras in the 24-hour period. The observed overall rate of error in this study was 6.2\% (27 episodes) and the overall rate of hesitation was 5.8\% (26 episodes). There was no statistically significant difference in error rates with the two hand washbasin configurations.

Conclusion: The amelioration of causes of error and hesitation by standardisation of the appearance and relative positioning of hand hygiene solutions at washbasins may translate to improved hand hygiene behaviours. Placement of moisturiser at the washbasin may not be essential. 


\section{Background}

Hand hygiene is well established as the fundamental principle and practice underscoring the prevention and control of healthcare-associated infection (HAI). ${ }^{1,2}$ Despite this, data from Hand Hygiene Australia (HHA) from 1 April 2014 to 30 June 2014, reports a mean hand hygiene compliance rate of emergency units in participating public and private hospitals as 73.9\% (95\% confidence interval [CI]: 73.3\%, 74.4\%). In comparison, mean compliance in medical and surgical units was 80.5\% (95\% CI: 80.2\%, 80.8\%) and 80.2\% (95\% CI: 80\%, 80.5\%) respectively. ${ }^{3}$ Poorer compliance in EDs has been attributed to a variety of factors, including time constraints, acuity of patient illness and injury, perceived urgency of the clinical situation, health worker skill mix, overcrowding, and the large numbers of patient contacts. $^{4-7}$

Improvement of healthcare workers (HCW) hand hygiene practices is therefore a challenge and multiple studies have indicated that hand hygiene compliance can only be improved and sustained through the use of an approach that takes into account the multifaceted nature of behaviour change. ${ }^{8-10}$ Efforts to improve hand hygiene compliance include continuous education and motivation programs ${ }^{9-12}$ and the measurement and feedback of hand hygiene compliance rate to users $2,9,10,12$

One important factor known to influence hand hygiene performance is the availability, utility and accessibility of hand hygiene products. ${ }^{2}$ Evidence and best practice indicates that in some instances of hand hygiene practice the location and accessibility of hand hygiene products directly influences hand hygiene behaviour. ${ }^{9-13}$ This is most notable with the accessibility of alcohol-based hand hygiene products. Put simply the closer the alcohol-based hand rub to the point of indication of use, such as the end of the patient bed or on the wall in each clinical 
care area, directly influences practice. Furthermore, research has shown that it saves time and

preliminary data reported by Widmer ${ }^{14,15}$ demonstrate better compliance than hand washing.

One author (MS) perceived a problem with the inability to identify and readily distinguish the different hand hygiene solutions located at hand washbasins in the ED. This resulted in his intermittent use of moisturising lotion instead of soap solution. This study sought to determine how frequent this problem was as measured by hesitation during product selection and use of an incorrect solution for hand washing.

\section{METHODS}

\section{Study Design}

A non-experimental, descriptive observational study was used to examine the hand hygiene practices of ED health personnel. The university and hospital human research ethics committees approved this study. There were no variations to the approved study protocol and no complaints received.

\section{Study Procedures}

\section{Study Setting and Population}

This study was conducted at a 760-bed metropolitan, adult, tertiary-referral hospital and level one trauma centre in Australia. The ED saw nearly 50,000 presentations annually with an admission rate of 35\%. In the acute and observation areas of the ED there were 21 hand washbasins of which 16 (defined as Type L) had the soap solution situated on the left of the basin and moisturising lotion on the right side of the basin. The remaining five basins (defined as Type R) had the reverse configuration with the moisturiser on the left and the 
soap solution on the right (see Figure 1). Alcohol-based hand hygiene product was available randomly throughout the unit in wall-mounted or stand-alone dispensers.

\section{Study Participants}

The participants in this study were staff of the ED. Written consent for participation was not sought due to the nature of the study and technology. Staff were aware the cameras were installed for observing hand hygiene practices but not the specific variables being measured. It was also impossible for staff to discern whether the cameras were filming once activated during use of the hand wash basins. Participant Information Sheets were emailed to all ED staff, posted on staff noticeboards, and circulated to all staff during meetings, two weeks prior to the commencement of the study to enable participant consent. Staff were advised that only six hand washbasins had cameras and they could use another basin if they did not want to participate in the study. While specific features such as type of uniform or presence of hospital identification tag were used to categorise the craft group from video footage, no person-identifiable information was recorded and no staff were generally identified or otherwise. To protect patient privacy, the cameras only had views of the hand washbasins and dispensers, and were positioned so that the cameras captured no facial features. With regard to staff privacy, the collected data did not include any subject identifying information and the video segments were not used to observe individual participants.

All ED staff (clinical and non-clinical) and visiting non-ED personnel (e.g. ambulance officers) working during the observation period in the ED were included in the study. Patients, visitors and individuals who could not be clearly identified as staff were excluded from the analyses. 


\section{Data Collection}

Six ceiling-mounted, motion-activated, electronic video cameras were installed above three Type L and three Type R washbasins on 12 November 2010. Hand hygiene practices were recorded over the next 18 days. Day 10 was selected for this study to minimise the Hawthorne effect associated with the participants' knowledge of camera installation. Data from a 24-hour period on day 10 of the recordings were collected into the Hand Hygiene Technique Observation Tool (HH-TOT) developed by authors JS and RS, based on Feldman's Criteria as modified by Larson and Lusk. ${ }^{16}$ This tool consisted of 24 variables, but only three were used in this analysis: (i) what product did the individual use to clean their hands; (ii) does the individual use an incorrect product for hand washing; and (iii) does the individual display hesitation during the selection of hand hygiene product (soap, alcohol or moisturiser). Responses for the variable-incorrect product, included whether the individual used moisturiser instead of soap, alcohol instead of soap, or water only (no solution). For the measurement of "hesitation", episodes were assessed as non-compliant if the individual moved to use one product, but stopped and used another, or applied one product and then changed to another.

The numbers of observations for the three variables were not exactly the same due to the inability to view some of the actions by participants because of camera angle, product placement, poor lighting and rapidity of hand movement.

Files equal to or less than 700 kilobytes (equating to less than 10 seconds) which were typically non-hand hygiene events (e.g. cleaning of basins), were excluded from the analysis 
unless they were linked with an event already in progress where the footage was separated into one or more files during filming.

\section{Data Analysis}

Descriptive statistical methods were used to organise, summarise and describe the data. Mean values were computed with standard deviation and median values with ranges. The numerator was based on the responses to the variable and the denominator, the overall number of hand hygiene episodes observed for each variable. Percentages were rounded to a single decimal point and may not total 100 for all variables because of rounding. All analyses were performed using SAS®.

\section{Results}

The majority of recorded episodes of hand hygiene were of ED staff $(n=424,92.4 \%)$. There were 29 (6.3\%) episodes by individuals who wore a staff uniform but were unable to be definitively identified as an ED nurse or doctor due to incomplete view of the participant or the image was too dark, however they were not patients or visitors. Only six (1.31\%) episodes were performed by non-ED staff e.g. ambulance officers (Table 1).

The six cameras recorded a total of 459 hand hygiene episodes in the 24-hour period. There were 171 (37.3\%) hand hygiene episodes recorded at Type L washbasins (soap solution on the left side and moisturiser on the right side) and 288 (62.7\%) at Type R washbasins (Table 1).

Analysis of product usage found that 412 (98.1\%) of intended hand hygiene episodes were performed correctly using a soap-based solution. Eight (1.9\%) episodes used an alcohol- 
based solution only. As alcohol should not be placed at the washbasin, according to guidelines, ${ }^{17}$ these were excluded from further analysis rather than trying to classify them as correct or incorrect episodes of hand hygiene (Table 2).

Table 3 shows that the correct product was used for 412 (93.8\%) hand hygiene episodes. Twenty-seven episodes (6.2\%) of hand hygiene episodes were performed incorrectly. For one episode (0.2\%), moisturiser was used instead of soap, one episode $(0.2 \%)$ used alcohol instead of soap, water only (without soap solution) was used for nine episodes (2.0\%), and 16 episodes (3.6\%) involved the use of two products simultaneously. The majority $(n=14$, 87.5\%) reached for moisturiser first and then dispensed soap solution.

Hesitation (Table 4) was observed for 26 hand hygiene episodes (5.8\%). For 11 episodes (2.4\%), the moisturiser was applied first and then soap solution; while in 15 (3.3\%) the potential error was recognised prior to application, and the hands were ultimately washed with soap solution.

Table 5 shows that moisturiser was only applied after hand washing for five (1.7\%) hand hygiene episodes. Another six episodes of moisturiser use, separate from a hand hygiene episode involving the use of soap and water, were also observed.

\section{Discussion}

Our study is novel in that it looks at a subgroup of people who are compliant with hand hygiene action but do actually wash their hands correctly. We found an overall error rate of 6.2\% for incorrect product use and 5.8\% for hesitation before using the correct product. We argue that the inconsistent placement of hand hygiene products contributes to poor hand 
hygiene behaviours, and that conversely standardising their relative positioning and colour/appearance would improve hand hygiene compliance. The standardisation of the relative location of hand wash solutions, such as soap on one side and hand drying agents on the other or preferably still locating moisturiser away from soap, may improve hand hygiene behaviours.

Lotions and creams are recommended to rehydrate (moisturise) and reduce damage to the skin from frequent hand cleansing activities. ${ }^{18}$ However our study found that only $1.7 \%$ of hand hygiene episodes used moisturiser at the hand washbasin. There were six instances where staff used the moisturiser at the basin as a specific event, separate from hand hygiene perse. The effect of the location of moisturiser on product selection should be examined, as it may be possible to move it away from a hand washbasin. The frequency of use of moisturiser alone was not reported, as such an episode may have been so short as to result in less than 700 kilobytes of data and as such would not have been analysed. However, it can be assumed that staff go to a hand washbasin to find moisturiser when they are not in the process of washing their hands do so as a learned response. As such it would be very easy for them to learn to go to another place in the department if it was moved. Colour coding would facilitate the speed of finding and learning the new locations and prevent confusion with the alcoholbased solution that is also placed away from the washbasin.

Although it is generally not recommended to position alcohol-based hand hygiene products at basins as the efficacy of alcohol is affected by a number of factors including whether the hands are wet when the alcohol is applied, some of the basins had the alcohol foam dispenser located at the basin. ${ }^{17}$ Therefore, these episodes where the alcohol-based solution was used correctly, without water, were excluded from the analysis. It may be that clinician was unable 
to easily identify and readily distinguish the different solutions at the washbasins.

Contributing to this may be the fact that the soap solution and the moisturiser were not being consistently positioned in relation to the basin in the ED (nor in other hospital departments, personal observation of MS) and being provided in visibly similar white containers.

The error rate reported here is for a sample only. Higher rates or error may occur for some staff, in particular nursing staff that comprises the largest workforce. As part of department practices, they are commonly assigned to work at a predetermined number of adjacent patient cubicles, and may use the same hand washbasin routinely and thus not be exposed to the different configurations of hand hygiene solutions at other basins in any particular shift.

\section{Study Limitations}

The inability to track individual staff member's frequency and pattern of use at the different types of hand washbasins may have underestimated the actual deleterious impact of lack of standardisation of means to identify and distinguish between the different hand hygiene products placed at a basin. The data used in the analyses came from day 10 of the recordings to ensure minimal change in behaviour as a consequence of staff being aware of the cameras. The study did not allow us to determine the relative contribution of the inconsistent position and lack of visible product differentiation to the observed error rates.

\section{Acknowledgements}

This research was funded by the Queensland Emergency Medicine Research Foundation (QEMRF-EMT-2008-009). 


\section{Conclusions}

The results of this study would support the need for standardisation of means to identify and distinguish between the different hand hygiene solutions found at or away from the hand washbasin. We would propose that at least two methods should be used to achieve this; both colour and placement. Standardisation of product identification will achieve a constant improvement in successful compliance with hand hygiene, removed from the vagaries of drop off rates seen with educational endeavours to improve compliance rates and will thus be cost effective to implement. 


\section{References}

1. Bjerke N. The Evolution. Hand washing to Hand Hygiene Guidance. Critical Care Nursing Quarterly. 2004;July-September, 27(3):295-307.

2. World Health Organization. WHO Guidelines on Hand Hygiene in Health Care. 2009: http://www.who.int/gpsc/en/ Accessed August 1, 2010.

3. Hand Hygiene Australia. National Data Set. 2014; http://www.hha.org.au/LatestNationalData.aspx.

4. Aacharya RP, Gastmans C, Denier Y. Emergency department triage: an ethical analysis. BMC Emergency Medicine. 2011;11(16). http://www.biomedcentral.com/1471-227X/11/16.

5. Al-Damouk M, Pudney E, Bleetman A. Hand hygiene and aseptic technique in the emergency department. Journal of Hospital Infection. 2004;56:137-141.

6. di Martino P, Ban KM, Bartoloni A, Fowler KE, Saint S, Mannelli F. Assessing the sustainability of hand hygiene adherence prior to patient contact in the emergency department: A 1-year postintervention evaluation. American Journal of Infection Control. 2011;39:14-18.

7. Meengs MR, Giles BK, Chisholm CD, Cordell WH, Nelson DR. Hand Washing Frequency in an Emergency Department. Annals of Emergency Medicine. 1994;23(6):1307-1312.

8. Creedon SA. Infection control: behavioural issues for healthcare workers. Clinical Governance: An International Journal. 2006;11(4):316-325.

9. Gould DJ, Moralejo D, Drey N, Chudleigh JH. Interventions to improve hand hygiene compliance in patient care. Cochrane Database of Systematic Reviews. 2010;8(9):CD005186.

10. Whitby M, Pessoa-Silva CL, McLaws M, et al. Behavioural considerations for hand hygiene practices: the basic building blocks. Journal of Hospital Infection. 2007;65(1):1-8.

11. Chou T, Kerridge J, Kulkarni M, Wickman K, Malow J. Changing the culture of hand hygiene compliance using a bundle that includes a violation letter. American Journal of Infection Control. 2010;38(7):575-578.

12. Moret L, Tequi B, Lombrail P. Should self-assessment methods be used to measure compliance with hand washing recommendations? A study carried out in a French university hospital. American Journal of Infection Control. 2004;32:384-390.

13. Bischoff W, Reynolds TM, Sessler CN, Edmond MB, Wenzel RP. Handwashing compliance by health care workers: The impact of introducing an accessible, alcohol-based hand antiseptic. Annals of Internal Medicine. 2000;160(7):1017-1021.

14. Widmer AF, Conselmann M, Tomic M, Frei R, Stranden AM. Introducing Alcohol-Based Hand Rub for Hand Hygiene: The Critical Need for Training. Infection Control and Hospital Epidemiology. 2007;28(1):50-54.

15. Widmer AF, Dangel M. Alcohol-Based Handrub: Evaluation of Technique and Microbiological Efficacy With International Infection Control Professionals. Infection Control and Hospital Epidemiology. 2004;25(3):207-209.

16. Larson E, Lusk E. Evaluating hand washing technique. Journal of Advanced Nursing. 1985;10:547-552.

17. Grayson ML, Russo P, Ryan K, Bellis K, Heard K. Hand Hygiene Australia. 5 Moments for Hand Hygiene. 2009: http://www.hha.org.au/. Accessed August 1, 2010.

18. Sedgewick J. Hand-washing in hospital wards. Nursing Times. 1984;80(20):64-67. 
Figure 1: Example of Type $L$ and Type $R$ Basins - as viewed by the clinician

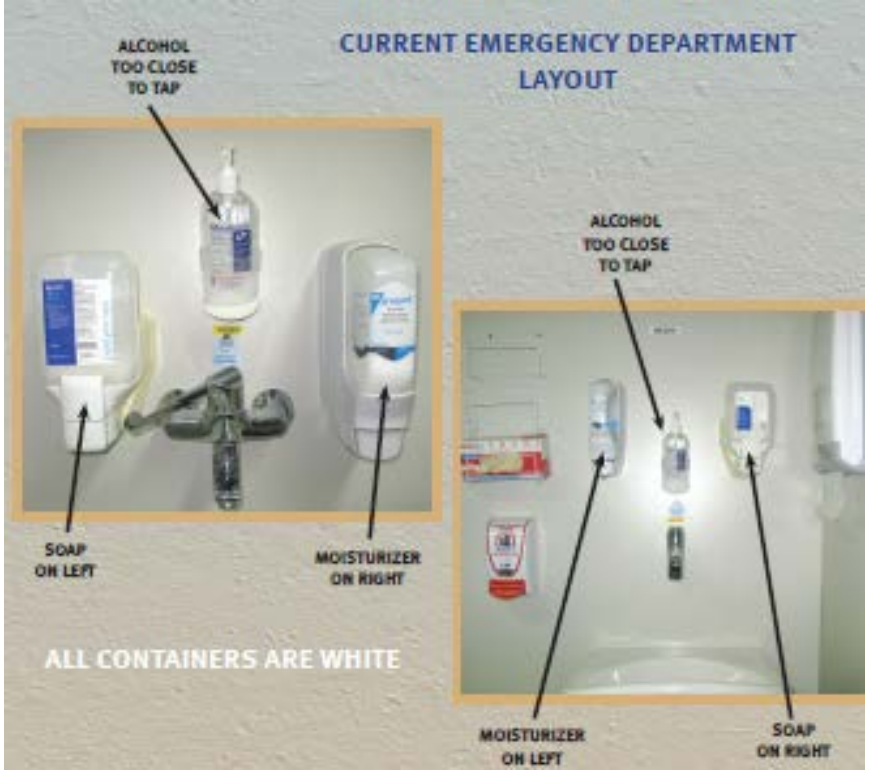

Figure 2: Hand Hygiene Basin Configuration as Viewed by Video Camera 2 (viewed right to left by clinician facing basin)



1. Paper towel dispenser

2. Soap dispenser

3. Hand wash basin

4. Sensor tap

5. Hand moisturiser dispenser

6. Glove holder

7. Alcohol-based hand foam dispenser

8. Waste receptacle (clinical)

NB green "2" sign refers to the actual washbasin number in the study 
Table 1. Frequencies for Professional Craft Groups

\begin{tabular}{|l|c|c|c|c|c|c|}
\hline \multirow{2}{*}{ Category } & \multicolumn{2}{|c|}{ Soap Left } & \multicolumn{2}{c|}{ Soap Right } & \multicolumn{2}{c|}{ Total } \\
\cline { 2 - 7 } & $\#$ & $\mathbf{\%}$ & $\#$ & $\mathbf{\%}$ & $\#$ & $\mathbf{\%}$ \\
\hline ED Staff & 167 & 97.7 & 257 & 89.2 & $\mathbf{4 2 4}$ & $\mathbf{9 2 . 4}$ \\
\hline Non-ED Staff & 1 & 0.6 & 5 & 1.7 & $\mathbf{6}$ & $\mathbf{1 . 3}$ \\
\hline $\begin{array}{l}\text { Unable to } \\
\text { determine }\end{array}$ & 3 & 1.8 & 26 & 9.0 & $\mathbf{2 9}$ & $\mathbf{6 . 3}$ \\
\hline Total & $\mathbf{1 7 1}$ & $\mathbf{3 7 . 3 \%}$ & $\mathbf{2 8 8}$ & $\mathbf{6 2 . 8 \%}$ & $\mathbf{4 5 9}$ & $\mathbf{1 0 0 \%}$ \\
\hline
\end{tabular}


Table 2. Frequencies for product use

\begin{tabular}{|l|c|c|c|c|c|c|}
\hline \multirow{2}{*}{ Category } & \multicolumn{2}{|c|}{ Soap Left } & \multicolumn{2}{c|}{ Soap Right } & \multicolumn{2}{c|}{ Total } \\
\cline { 2 - 7 } & $\#$ & $\%$ & $\#$ & $\%$ & $\#$ & $\mathbf{\%}$ \\
\hline Soap-based wash & 160 & 99.4 & 252 & 97.3 & $\mathbf{4 1 2}$ & $\mathbf{9 8 . 1}$ \\
\hline $\begin{array}{l}\text { Alcohol-based } \\
\text { foam }\end{array}$ & 1 & 0.6 & 7 & 2.7 & $\mathbf{8}$ & $\mathbf{1 . 9}$ \\
\hline Total & $\mathbf{1 6 1}$ & $\mathbf{3 8 . 3}$ & $\mathbf{2 5 9}$ & $\mathbf{6 1 . 7}$ & $\mathbf{4 2 0}$ & $\mathbf{1 0 0}$ \\
\hline
\end{tabular}

Footnote: The numbers of observations for the three variables were not exact due to the inability to view some of the actions by participants because of camera angle, product placement, poor lighting and rapidity of hand movement.

Table 3. Frequency for Use of Incorrect Product

\begin{tabular}{|l|c|c|c|c|c|c|}
\hline \multirow{2}{*}{ Category } & \multicolumn{2}{|c|}{ Left } & \multicolumn{2}{c|}{ Right } & \multicolumn{2}{c|}{ Total } \\
\cline { 2 - 7 } & $\#$ & $\%$ & $\#$ & $\%$ & $\#$ & \% \\
\hline Used Correctly & 160 & 95.2 & 252 & 93.0 & $\mathbf{4 1 2}$ & $\mathbf{9 3 . 8}$ \\
\hline $\begin{array}{l}\text { Moisturiser used } \\
\text { instead of soap }\end{array}$ & 0 & - & 1 & 0.4 & $\mathbf{1}$ & $\mathbf{0 . 2}$ \\
\hline $\begin{array}{l}\text { Alcohol-based } \\
\text { foam with water }\end{array}$ & 0 & - & 1 & 0.4 & $\mathbf{1}$ & $\mathbf{0 . 2}$ \\
\hline $\begin{array}{l}\text { Water only used } \\
\text { and no solution }\end{array}$ & 2 & 1.2 & 7 & 2.6 & $\mathbf{9}$ & $\mathbf{2 . 1}$ \\
\hline $\begin{array}{l}\text { Two products } \\
\text { used } \\
\text { simultaneously } \\
\text { e.g. soap and } \\
\text { moisturiser }\end{array}$ & 6 & 3.6 & 10 & 3.7 & $\mathbf{1 6}$ & $\mathbf{3 . 6}$ \\
\hline $\begin{array}{l}\text { Subtotal } \\
\text { Incorrect }\end{array}$ & 8 & 4.8 & 19 & 7.0 & $\mathbf{2 7}$ & $\mathbf{6 . 2}$ \\
\hline Total & $\mathbf{1 6 8}$ & $\mathbf{3 8 . 3}$ & $\mathbf{2 7 1}$ & $\mathbf{6 1 . 7}$ & $\mathbf{4 3 9}$ & $\mathbf{1 0 0}$ \\
\hline
\end{tabular}

Footnote: The numbers of observations for the three variables were not exact due to the inability to view some of the actions by participants because of camera angle, product placement, poor lighting and rapidity of hand movement. 
Table 4. Frequencies for Hesitation During Product Selection

\begin{tabular}{|l|c|c|c|c|c|c|}
\hline \multirow{2}{*}{ Category } & \multicolumn{2}{|c|}{ Left } & \multicolumn{2}{c|}{ Right } & \multicolumn{2}{c|}{ Total } \\
\cline { 2 - 7 } & $\#$ & $\%$ & $\#$ & $\%$ & $\#$ & \% \\
\hline $\begin{array}{l}\text { Did not hesitate, } \\
\text { immediately } \\
\text { selected hand } \\
\text { hygiene product }\end{array}$ & 158 & 93.5 & 255 & 90.7 & $\mathbf{4 1 3}$ & $\mathbf{9 1 . 8}$ \\
\hline $\begin{array}{l}\text { Moved to use } \\
\text { one product, but } \\
\text { stopped and used } \\
\text { another }\end{array}$ & 5 & 3.0 & 10 & 3.6 & $\mathbf{1 5}$ & $\mathbf{3 . 3}$ \\
\hline $\begin{array}{l}\text { Applied one } \\
\text { product and then } \\
\text { changed to } \\
\text { another }\end{array}$ & 4 & 2.4 & $\mathbf{7}$ & 2.5 & $\mathbf{1 1}$ & $\mathbf{2 . 4}$ \\
\hline Sub-Total (error) & 9 & 5.3 & 17 & 6.0 & $\mathbf{2 6}$ & $\mathbf{5 . 8}$ \\
\hline Total & $\mathbf{1 6 9}$ & $\mathbf{3 7 . 6}$ & $\mathbf{2 8 1}$ & $\mathbf{6 2 . 4}$ & $\mathbf{4 5 0}$ & - \\
\hline
\end{tabular}

Footnote: The numbers of observations for the three variables were not exact due to the inability to view some of the actions by participants because of camera angle, product placement, poor lighting and rapidity of hand movement. 
Table 5: Frequencies for Application of Hand Moisturiser (variable examined at 4 hand wash basins only, including basin 2)

\begin{tabular}{|l|c|c|}
\hline Category & $\#$ & $\mathbf{\%}$ \\
\hline No hand moisturiser used after washing & 283 & $\mathbf{9 8 . 3}$ \\
\hline Yes hand moisturiser used after washing & 5 & $\mathbf{1 . 7}$ \\
\hline Sub-Total (related to hand washing) & $\mathbf{2 8 8}$ & $\mathbf{1 0 0}$ \\
\hline Hand moisturiser used without washing & 6 & - \\
\hline Total & $\mathbf{2 9 4}$ & $\mathbf{2 . 0}$ \\
\hline
\end{tabular}

